\title{
Serum Selenium Levels in Patients with Graves Disease: Associations with Clinical Activity and Severity in a Retrospective Case-control Study
}

\author{
Tae Hwan Kim¹, JaeSang Koํㅡ, Bo Ram Kim¹, Dong Yeob Shin², Eun Jig Lee², Jin Sook Yoon ${ }^{1}$ \\ ${ }^{1}$ Department of Ophthalmology, Institute of Vision Research, Yonsei University College of Medicine, Seoul, Korea \\ ${ }^{2}$ Division of Endocrinology and Metabolism, Department of Internal Medicine, Yonsei University College of Medicine, Seoul, Korea
}

Purpose: To compare serum selenium levels in Graves patients and non-Graves control participants and to evaluate associations between serum selenium levels and clinical features of Graves orbitopathy (GO).

Methods: We conducted a single-center, retrospective case-control study among 33 patients with Graves disease without GO (GD), 31 patients with diagnosed GO, and 27 unaffected healthy participants enrolled between 2013 and 2020 at Severance Hospital. We compared serum selenium concentrations between the GD, GO, and healthy control groups, and analyzed associations between serum selenium and GO patients' clinical activity scores, severity (assessed through modified NOSPECS scores), and other clinical features using multivariate linear regression analysis.

Results: Mean serum selenium levels were $109.30 \pm 16.39,111.39 \pm 14.04$, and $126.09 \pm 21.09 \mathrm{ng} / \mathrm{mL}$ in GO patients, GD patients, and healthy control participants, respectively. Mean serum selenium levels in Graves patients with and without orbitopathy were significantly lower than those in the healthy control group $(p<0.05)$, and mean selenium levels were slightly lower in GO than those in GD patients $(p=0.594)$. Serum selenium levels were significantly lower in GO patients with eyelid retraction than in patients without retraction $(p=0.038)$. However, serum selenium levels were not associated with clinical activity scores and modified NOSPECS scores ( $p=0.241$ and 0.801 , respectively).

Conclusions: Serum selenium levels were significantly lower in Graves patients with or without GO, compared to non-Graves control participants. Selenium levels were not associated with clinical activity scores or NOSPECS scores, though we observed an association with eyelid retraction.

Key Words: Eyelids, Inflammation, Orbit, Selenium

Graves orbitopathy (GO) is an autoimmune inflammatory disease of the orbit and periorbital tissues that occurs in

Received: September 16, 2021 Final revision: October 19, 2021 Accepted: October 29, 2021

Corresponding Author: Jin Sook Yoon, MD, PhD. Department of Ophthalmology, Institute of Vision Research, Yonsei University College of Medicine, 50 Yonsei-ro, Seodaemun-gu, Seoul 03722, Korea. Tel: 82-22228-3570, Fax: 82-2-312-0541, E-mail: yoonjs@yuhs.ac approximately $20 \%$ to $50 \%$ of patients with Graves disease [1]. GO is characterized by eyelid retraction and swelling, redness, exophthalmos, and diplopia. These clinical features can cause significant sequelae if appropriate treatment is not initiated in the early stages of the disease. The pathogenesis of GO is yet to be clarified, though the activation of orbital fibroblasts is known to play a key role in the development of the disease.

Selenium is an essential trace element that is an important 
component of several major metabolic pathways, such as thyroid hormone metabolism and immune function. Several epidemiological studies have reported that low selenium status is linked to an increased prevalence of benign thyroid disease, including Graves disease and thyroiditis [2-4]. As a result, oral selenium administration has become increasingly prevalent in the medical treatment of thyroid disease.

A prospective randomized trial revealed that selenium administration significantly reduced ocular involvement, improved quality of life, and reduced disease progression in mild GO patients [5]. Based on this study, the European Thyroid Association/European Group on Graves Orbitopathy recommend a 6-month period of selenium supplementation in patients with mild GO of short duration [6]. However, there are some limitations to the aforementioned study. First, the baseline serum selenium level was not measured prior to oral selenium supplementation. Therefore, it is not known whether correcting selenium deficiency (vs. raising selenium levels with or without a deficiency) improved ocular involvement. An additional concern is that it is difficult to ascertain how serum selenium levels relate to clinical symptoms of GO, even within gold-standard randomized prospective trials. Second, selenium deficiency is common in Europe, where the study was conducted. However, selenium intake is generally high in North America and East Asia [7,8]. Hence, it is unclear whether selenium supplementation would have the same therapeutic effect in GO patients within countries where selenium deficiency is relatively rare. In fact, the American Thyroid Association has not endorsed the European guidelines for oral selenium supplementation due to these regional differences [9].

In this study, we investigated serum selenium levels among patients with Graves disease while comparing them to nonGraves healthy control participants. Our study was conducted in South Korea, where selenium intake is relatively high $[10,11]$. We also attempted to examine associations between serum selenium levels and clinical features of GO.

\section{Materials and Methods}

\section{Patients}

This study was conducted among patients with Graves disease who initially visited both Departments of Endocri- nology and Ophthalmology at Severance Hospital at the Yonsei University College of Medicine from 2013 to 2020. These participants were retrospectively enrolled in our study. The healthy control group was comprised of participants with selenium blood tests conducted during an evaluation for antioxidant capacity at the Severance health check-up center. This study was approved by the institutional review board of the Yonsei University College of Medicine (No. 4-2020-1222). Informed consent was waived because of minimal risk of personal information. The study was conducted in accordance with the provisions of the Declaration of Helsinki.

This study included 33 patients with Graves disease without GO (GD), 31 patients with GO, and 27 healthy control participants. The controls were age-matched with patients participating in the study. We abstracted demographic and medical information, including sex, age, treatment of Graves disease, and other medical history, from hospital medical records. Thyroid function tests, including thyroid stimulating hormone, tri-iodothyronine, free thyroxine, and thyroid stimulating hormone receptor antibody levels, were conducted using archived blood samples. All patients had been examined for clinical ophthalmologic symptoms by one ophthalmologist (JSY) using the following assessments: slit-lamp examination, visual acuity, intraocular pressure, exophthalmometry measured with a Hertel exophthalmometer, the Hess screen test, the binocular single vision test, and an orbital computed tomography scan.

\section{Clinical Graves disease orbitopathy evaluation}

GO clinical activity was assessed using the clinical activity score (CAS), as described by Mourits et al. [12]. There are seven signs of inflammation of the orbit: spontaneous retrobulbar pain, pain during eye movement, eyelid erythema, conjunctival injection, chemosis, swelling of the lacrimal caruncle, and eyelid oedema or fullness. The CAS grading scale ranges from 0 to 7 . GO patients were divided into two groups according to CAS grade: 0 to 2 and 3 to 7 , corresponding to inactive and active disease, respectively.

The severity of GO was evaluated through a modified NOSPECS score based on each of these symptoms: soft-tissue involvement, proptosis, extraocular muscle involvement, lid retraction, site difference, corneal defect, and optic nerve compression [13], and scored on a scale of 
0 to 17. Patients were divided into two groups based on the modified NOSPECS scores: 0 to 4 , and 5 to 17 , which correspond to mild and moderate to severe disease, respectively.

\section{Determination of serum selenium levels}

Blood samples were centrifuged for 15 minutes at 2,500 $\mathrm{rpm}$. Serum was separated into acid-washed tubes and stored at $-20^{\circ} \mathrm{C}$ until being thawed at room temperature for analysis. A working standard solution $(10 \mu \mathrm{g} / \mathrm{mL})$ was prepared from a $1 \mathrm{mg} / \mathrm{mL}$ selenium stock solution (SPEX CertiPrep, Metuchen, NJ, USA). During the analysis, all serum samples and working solutions were prepared by dilution $(1: 19, \mathrm{v} / \mathrm{v})$ in an acidic solution $(1 \% \mathrm{HNO}, 0.01$ mM EDTA, 0.07\% Triton X-100, 1.5\% 1-butanol) [14]. Serum selenium level measurement was conducted through automated inductively coupled plasma mass spectrometry technology, using an Elan DRC-II apparatus (Perkin-Elmer, Waltham, MA, USA). The results are expressed as nanograms per milliliter of serum (ng/mL).

\section{Statistical analysis}

All statistical analyses were performed using IBM SPSS ver. 19.0 (IBM Corp., Armonk, USA). Data were expressed as mean values with respective standard deviations. The normal distribution of the data was confirmed using the Kolmogorov-Smirnov test. Categorical data were analyzed using the chi-square test. For the non-parametric data, the Kruskal-Wallis test was used to perform statistical comparisons among three groups, and the Mann-Whitney $U$-test was used to perform statistical comparisons between two groups. Independent $t$-tests were used for the comparison of serum selenium levels between the mild and moderate to severe GO groups. Associations between serum selenium levels and the clinical features of GO were analyzed using Pearson correlation and multiple regression analysis. Statistical significance was defined as $p<0.05$.

\section{Results}

This study retrospectively enrolled 91 participants. To analyze differences in serum selenium status among GD and GO patients, participants were divided into three groups: a healthy group of control participants $(n=27)$, the GD group ( $\mathrm{n}=33)$, and the GO group $(\mathrm{n}=31)$. Participants' baseline characteristics are summarized in Tables 1 and 2 . Of the enrolled participants, 72 (79.1\%) were female and $19(20.9 \%)$ were male.

Mean serum selenium levels were $109.30 \pm 16.39 \mathrm{ng} / \mathrm{mL}$ in GO patients, $111.39 \pm 14.04 \mathrm{ng} / \mathrm{mL}$ in GD patients, and $126.09 \pm 21.09 \mathrm{ng} / \mathrm{mL}$ in the healthy control group. Mean

Table 1. Clinical characteristics of the study population

\begin{tabular}{|c|c|c|c|c|}
\hline Characteristics & $\mathrm{GO}(\mathrm{n}=31)$ & $\mathrm{GD}(\mathrm{n}=33)$ & Control $(\mathrm{n}=27)$ & $p$-value \\
\hline Age (yr) & $36.96 \pm 13.21$ & $38.21 \pm 8.33$ & $35.00 \pm 8.63$ & 0.39 \\
\hline Sex (male : female) & $4: 27$ & $7: 26$ & $8: 19$ & 0.29 \\
\hline Disease duration of Graves disease (mon) & $34.78 \pm 48.79$ & $28.36 \pm 33.44$ & - & 0.32 \\
\hline Disease duration of GO (mon) & $24.94 \pm 40.98$ & - & - & - \\
\hline Thyroid stimulating hormone $(\mu \mathrm{IU} / \mathrm{mL})$ & $1.04 \pm 2.22$ & $0.79 \pm 1.51$ & - & 0.30 \\
\hline Tri-iodothyronine $(\mathrm{ng} / \mathrm{mL})$ & $1.86 \pm 1.46$ & $2.16 \pm 2.01$ & & 0.23 \\
\hline Free thyroxine (ng/dL) & $1.62 \pm 1.22$ & $1.76 \pm 0.89$ & - & 0.30 \\
\hline Anti-thyroid stimulating hormone antibody (IU/L) & $13.53 \pm 14.65$ & $11.45 \pm 13.92$ & - & 0.28 \\
\hline Clinical activity score (range, $0-6$ ) & $2.00 \pm 1.46$ & - & - & - \\
\hline Clinical severity score (modified NOSPECS, $0-17$ ) & $4.67 \pm 2.66$ & - & - & - \\
\hline Serum selenium level (ng/mL) & $109.30 \pm 16.39$ & $111.39 \pm 14.04$ & $126.09 \pm 21.09$ & $<0.05^{*}$ \\
\hline
\end{tabular}

Values are presented as mean \pm standard deviation or number; Continuous variables were assessed with Kruskal-Wallis tests and MannWhitney $U$-test, while categorical data (sex) were assessed with chi-square test.

$\mathrm{GO}=$ Graves orbitopathy; GD = Graves disease without GO

*A $p$-value less than 0.05 was considered statistically significant. 
Table 2. Characteristics of Graves disease with orbitopathy patients with ocular manifestations

\begin{tabular}{lc}
\hline Ocular manifestation & No. of patients $(\%)$ \\
\hline Proptosis $(\mathrm{mm})$ & $5(16.1)$ \\
$<17$ & $7(22.6)$ \\
$17-18$ & $12(38.7)$ \\
$19-22$ & $7(22.6)$ \\
$>22$ & $12(38.7)$ \\
Diplopia & $14(45.1)$ \\
Extraocular muscle enlargement & $14(45.1)$ \\
Lid retraction & $3(9.7)$ \\
Compressive optic neuropathy & \\
\hline
\end{tabular}

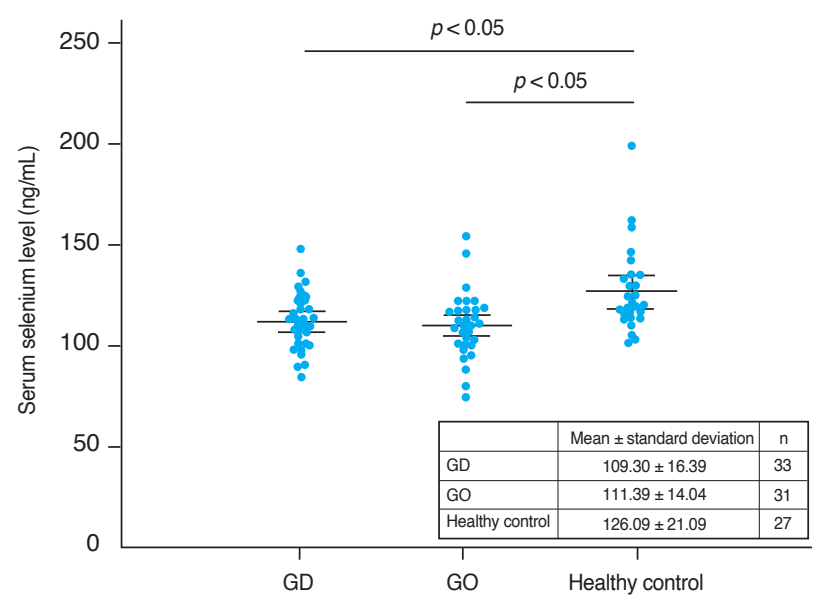

Fig. 1. Comparison between the mean selenium levels in the study groups. Serum selenium levels in Graves disease without Graves orbitopathy (GO) (GD) and with GO patients were significantly lower than in the healthy group (each $p<0.05$ ). Mean selenium levels were lower in GO than in GD patients, but this difference was not statistically significant $(p=0.594)$. The results are expressed as the mean \pm standard deviation. Error bars represent standard deviation.

serum selenium levels in GD and GO patients were significantly lower than in the healthy group (each $p<0.05$ ) (Fig. 1). Mean selenium levels were lower in GO than in GD patients, but this difference was not statistically significant ( $p$ $=0.594$ ).

The mean CAS and modified NOSPECS score among GO patients were $2.00 \pm 1.46$ and $4.67 \pm 2.66$, respectively. We examined the serum selenium status among GO patients who were categorized into an inactive group (CAS, 0-2) and an active group (CAS, 3-7), and into a mild group and a moderate to severe group according to clinical severity. The mean serum selenium level was higher in the

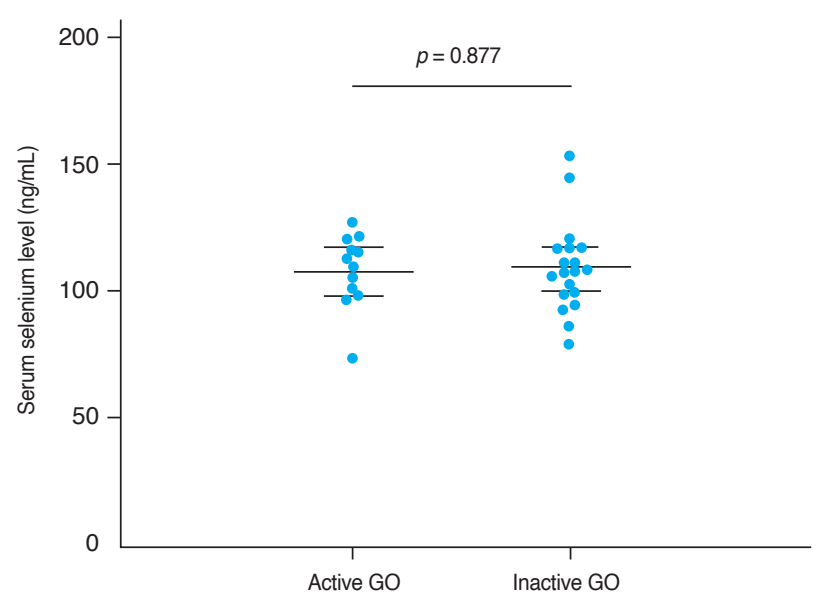

Fig. 2. Comparison of selenium status by Graves orbitopathy (GO) clinical activity. GO clinical activity was assessed using the clinical activity score (CAS). The CAS grading scale ranges from 0 to 7. GO patients were divided into two groups according to CAS grade: 0 to 2, and 3 to 7 , corresponding to inactive and active GO disease, respectively. The mean serum selenium level was higher in the inactive GO group $(109.67 \pm 17.80 \mathrm{ng} / \mathrm{mL})$ than in the active GO group $(108.71 \pm 14.63 \mathrm{ng} / \mathrm{mL})$, but this difference was not statistically significant $(p=0.877)$. Error bars represent standard deviation.

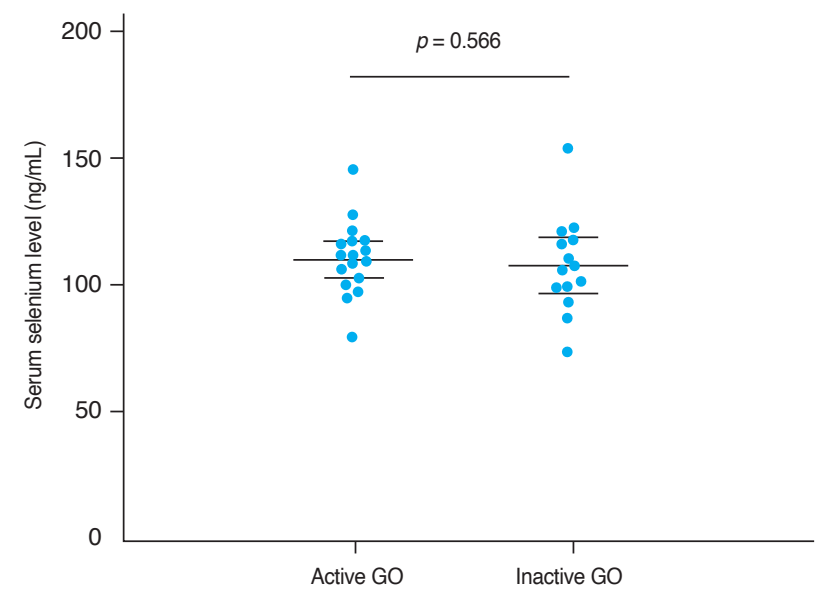

Fig. 3. Comparison of selenium status by Graves orbitopathy (GO) severity. GO clinical severity was evaluated using assessed using the modified NOSPECS score. The modified NOSPECS score ranges from 0 to 17. Patients were divided into two groups based on the modified NOSPECS scores: 0 to 4 , and 5 to 17 , which correspond to mild and moderate to severe disease, respectively. The mean serum selenium level was higher in the mild GO group $(110.52 \pm 14.42 \mathrm{ng} / \mathrm{mL})$ than in the moderate to severe GO group $(107.82 \pm 18.97 \mathrm{ng} / \mathrm{mL})$, but this difference was not statistically significant $(p=0.566)$. Error bars represent standard deviation. 
Table 3. Linear regression analysis for the association of serum selenium level with clinical and biochemical factors of Graves orbitopathy

\begin{tabular}{|c|c|c|c|c|}
\hline \multirow{2}{*}{ Factor } & \multicolumn{2}{|c|}{ Univariate } & \multicolumn{2}{|c|}{ Multivariate } \\
\hline & Beta & $p$-value & Beta & $p$-value \\
\hline Sex & 0.436 & $0.014^{*}$ & 0.241 & 0.155 \\
\hline Age & 0.042 & 0.822 & -0.050 & 0.785 \\
\hline Disease duration & -0.129 & 0.496 & - & - \\
\hline Tri-iodothyronine & 0.008 & 0.967 & - & - \\
\hline Free thyroxine & -0.343 & 0.059 & -0.324 & 0.066 \\
\hline Anti-thyroid stimulating hormone receptor antibody & -0.030 & 0.872 & - & - \\
\hline Visual acuity & 0.081 & 0.664 & - & - \\
\hline Intraocular pressure & -0.256 & 0.165 & - & - \\
\hline Clinical activity score & 0.068 & 0.716 & 0.243 & 0.241 \\
\hline Modified NOSPECS & -0.100 & 0.592 & 0.062 & 0.801 \\
\hline Proptosis & 0.112 & 0.549 & - & - \\
\hline Diplopia & -0.043 & 0.818 & - & - \\
\hline Eyelid retraction & -0.425 & $0.017^{*}$ & -0.396 & $0.038^{*}$ \\
\hline Extraocular muscle enlargement & 0.060 & 0.749 & - & - \\
\hline Compressive optic neuropathy & -0.212 & 0.253 & -0.330 & 0.114 \\
\hline
\end{tabular}

*A $p$-value less than 0.05 was considered statistically significant.

inactive group $(109.67 \pm 17.80 \mathrm{ng} / \mathrm{mL})$ than in the active group $(108.71 \pm 14.63 \mathrm{ng} / \mathrm{mL})$ and was higher in the mild group $(110.52 \pm 14.42 \mathrm{ng} / \mathrm{mL})$ than in the moderate to severe group $(107.82 \pm 18.97 \mathrm{ng} / \mathrm{mL})$, though these differences were not statistically significant ( $p=0.877$ and 0.656 , respectively) (Fig. 2, 3).

Univariate analyses of GO clinical manifestations with respect to serum selenium levels were conducted using a linear regression model; sex (beta $=-0.436, p=0.014$ ) and eyelid retraction (beta $=-0.425, p=0.017$ ) were significantly related to serum selenium level. Multivariable analysis indicated that eyelid retraction showed a significant negative correlation with serum selenium level $($ beta $=$ $-0.396, p=0.038$ ) (Table 3).

\section{Discussion}

Since the publication of a multicenter, placebo controlled, randomized study on the effects of short-term oral selenium supplementation in patients with mild GO in a European population in 2011, oral selenium supplementation therapy has become globally popular $[5,6]$. In fact, al- most all responders (94\%) within a survey of European Thyroid Association members recommended selenium supplementation for GO patients [15].

Several observational studies have also been conducted to determine the relationship between GO and selenium. Khong et al. [16] revealed that serum selenium was lower in patients with GO than in those without GO and suggested that selenium deficiency could be a risk factor for clinical manifestation of GO in GD patients. Dehina et al. [17] also confirmed that serum selenium levels in GO were lower than in healthy control participants but did not demonstrate an association between selenium levels and GO severity.

In this study, we compared serum selenium status between GD patients, GO patients, and normal healthy control participants presenting at a hospital in South Korea, where selenium intake is relatively high. We also explored associations between clinical manifestations of GO and serum selenium levels in GO patients. The mean serum selenium level among the healthy control participants was $126.09 \pm 21.09 \mathrm{ng} / \mathrm{mL}$. This value did not differ significantly from those previously reported in Korea [10,11]. These results reaffirm previous findings of abundant selenium 
intake in the Korean population, similar to that of the United States and Japanese populations [18].

Selenium is an important component of antioxidant enzymes, such as glutathione peroxidase (GPx), thioredoxin reductase, and iodothyronine deiodinase [19]. A number of recent studies have demonstrated that oxidative stress may play a role in the pathogenesis of GO [20,21]. Overproduction of reactive oxygen species (ROS) resulting from an imbalance in the antioxidant system is known to have a major impact on the development of GO, by promoting the proliferation of orbital fibroblasts and the release of glycosaminoglycans, as well as the secretion of inflammatory cytokines and the expression of other factors (heat shock protein-72, human leukocyte antigen-DR, intercellular adhesion molecule-1) involved in the expression of GO [21]. The human thyroid gland is known to contain the highest amount of selenium per gram of tissue than any other body organ, and selenium plays an important role in the removal of excessive ROS derived from thyroid metabolism [22]. Thus, removing excessively generated ROS in Graves disease requires more selenium than the necessary amount among healthy participants, and hence lower serum selenium levels may be observed among GD and GO participants compared to healthy control participants. This decreased level of selenium caused by the consumption of selenium to relieve oxidative stress in the body is observed not only in Graves disease but also in other systemic diseases that cause an increase in oxidative stress [23,24].

It is noteworthy that serum selenium levels in GD and GO patients in this South Korean study were even higher than those of normal healthy people in European studies [7,8]. This result raises questions about whether treatment with oral selenium supplementation will be effective in patients with GD and GO in areas with relatively high serum levels of selenium due to sufficient selenium intake. The activity of GPx, which plays a major role in the antioxidant effect among selenoproteins, is generally known to increase as the concentration of selenium increases. However, several studies have shown that GPx activity is no longer significant if the level of serum selenium increases above a certain level [25-27]. Based on these studies, the concentration of serum selenium required for optimal plasma GPx activity is reported to be $100 \mathrm{ng} / \mathrm{mL}[27,28]$. In other words, previous studies conducted in Europe showed that the majority of patients did not meet the requirements for optimal GPx activity due to low serum se- lenium levels, necessitating oral selenium supplementation to improve clinical manifestations of GO. However, as shown in this study, oral selenium supplementation may not have a significant effect on GPx activity or may not be effective in improving GO clinical symptoms in certain populations. Furthermore, excessive selenium intake may cause other complications, such as diabetes [29]. Hence, measuring blood levels of selenium might be helpful prior to recommending oral selenium supplementation in a medical setting, and further clinical trials are needed to inform objective selenium intake guidelines for GO patients in populations with sufficient selenium intake, weighing the risks and benefits of therapeutic supplementation.

We observed no significant correlation between serum selenium levels and the clinical activity and severity of GO in this study, with the exception of the finding that lower serum selenium concentrations were associated with eyelid retraction. In a multicenter placebo controlled clinical trial, a significant improvement in eyelid retraction was apparent in the selenium supplementation group for mild GO patients [5]; however, it is not yet clear how selenium affects this GO phenotype. Muller's muscle is a smooth muscle adjoining the levator palpebrae muscle that helps elevate the upper eyelid; this muscle is controlled by the sympathetic nervous system [30]. In GO patients, eyelid retraction can occur because of hyperactivity of the superior tarsal muscle due to overactivity of the sympathetic nervous system [31,32]. Eyelid retraction could also occur because of infiltration of macrophages and mast cells into Muller's muscle, causing adhesion between Muller's muscle and the surrounding tissue [31]. Smith and Douglas [33] speculated that selenium might influence the sympathetic tone of the ocular muscles as well as reducing inflammation of the Muller's muscle. Several existing studies have reported that ROS can stimulate sympathetic nervous system activity, thereby affecting systemic diseases [34,35], and ROS generated by hypermetabolism in Graves disease may induce eyelid retraction through activation of the sympathetic nervous system. If the selenium level in the body is sufficient to produce an optimal antioxidant effect, it could relieve sympathetic nerve overactivity by reducing oxidative stress, though there is no mechanistic evidence to prove its therapeutic value in the reduction of eyelid retraction.

A substantial strength of this study is that it was conducted in areas with relatively sufficient selenium intakes, 
while most previous studies were conducted in areas with insufficient selenium intake. However, we acknowledge several limitations of this study, including its relatively small sample size, measuring selenium levels at one time point only, and using serum selenium level as the sole indicator of selenium status in the body.

In summary, we found that selenium levels among GD and GO patients were significantly lower than among healthy participants in a study conducted in South Korea. However, serum selenium was not associated with GO activity and severity, except for eyelid retraction. Further studies are needed to provide evidence for the effectiveness of oral selenium supplementation in GO patients presenting within selenium-abundant populations, as well as evidence of how selenium affects the clinical features of GO.

\section{Conflicts of Interest}

No potential conflict of interest relevant to this article was reported.

\section{Acknowledgements}

None.

\section{Funding}

This work was supported by the National Research Foundation of Korea (NRF) grant funded by the Korea government (MSIT) (No. 2021R1F1A104665211).

\section{References}

1. Hiromatsu Y, Eguchi H, Tani J, et al. Graves' ophthalmopathy: epidemiology and natural history. Intern Med 2014;53:353-60.

2. Wu Q, Rayman MP, Lv H, et al. Low population selenium status is associated with increased prevalence of thyroid disease. J Clin Endocrinol Metab 2015;100:4037-47.

3. Rayman MP. Multiple nutritional factors and thyroid disease, with particular reference to autoimmune thyroid dis- ease. Proc Nutr Soc 2019;78:34-44.

4. Wichman J, Winther KH, Bonnema SJ, Hegedus L. Selenium supplementation significantly reduces thyroid autoantibody levels in patients with chronic autoimmune thyroiditis: a systematic review and meta-analysis. Thyroid 2016;26:1681-92.

5. Marcocci C, Kahaly GJ, Krassas GE, et al. Selenium and the course of mild Graves' orbitopathy. $N$ Engl J Med 2011;364:1920-31.

6. Bartalena L, Baldeschi L, Boboridis K, et al. The $2016 \mathrm{Eu}-$ ropean Thyroid Association/European Group on Graves' Orbitopathy Guidelines for the management of Graves' orbitopathy. Eur Thyroid J 2016;5:9-26.

7. Johnson CC, Fordyce FM, Rayman MP. Symposium on 'Geographical and geological influences on nutrition': factors controlling the distribution of selenium in the environment and their impact on health and nutrition. Proc Nutr Soc 2010;69:119-32.

8. Stoffaneller R, Morse NL. A review of dietary selenium intake and selenium status in Europe and the Middle East. Nutrients 2015;7:1494-537.

9. Ross DS, Burch HB, Cooper DS, et al. 2016 American Thyroid Association Guidelines for diagnosis and management of hyperthyroidism and other causes of thyrotoxicosis. Thyroid 2016;26:1343-421.

10. Kim YJ, Galindev O, Sei JH, et al. Serum selenium level in healthy Koreans. Biol Trace Elem Res 2009;131:103-9.

11. Kim IW, Bae SM, Kim YW, et al. Serum selenium levels in Korean hepatoma patients. Biol Trace Elem Res 2012;148:25-31.

12. Mourits MP, Prummel MF, Wiersinga WM, Koornneef L. Clinical activity score as a guide in the management of patients with Graves' ophthalmopathy. Clin Endocrinol (Oxf) 1997;47:9-14.

13. Eckstein AK, Plicht M, Lax H, et al. Thyrotropin receptor autoantibodies are independent risk factors for Graves' ophthalmopathy and help to predict severity and outcome of the disease. J Clin Endocrinol Metab 2006;91:3464-70.

14. Sohn OS, Blackwell L, Mathis J, et al. Excretion and tissue distribution of selenium following treatment of male F344 rats with benzylselenocyanate or sodium selenite. Drug Metab Dispos 1991;19:865-70.

15. Negro R, Hegedus L, Attanasio R, et al. A 2018 European Thyroid Association Survey on the use of selenium supplementation in Graves' hyperthyroidism and Graves' orbitopathy. Eur Thyroid J 2019;8:7-15. 
16. Khong JJ, Goldstein RF, Sanders KM, et al. Serum selenium status in Graves' disease with and without orbitopathy: a case-control study. Clin Endocrinol (Oxf) 2014;80:905-10.

17. Dehina N, Hofmann PJ, Behrends T, et al. Lack of association between selenium status and disease severity and activity in patients with Graves' ophthalmopathy. Eur Thyroid $J$ 2016;5:57-64.

18. Winther KH, Rayman MP, Bonnema SJ, Hegedus L. Selenium in thyroid disorders: essential knowledge for clinicians. Nat Rev Endocrinol 2020;16:165-76.

19. Tinggi U. Selenium: its role as antioxidant in human health. Environ Health Prev Med 2008;13:102-8.

20. Lanzolla G, Marcocci C, Marino M. Oxidative stress in graves disease and graves orbitopathy. Eur Thyroid $J$ 2020;9(Suppl 1):40-50.

21. Bartalena L, Tanda ML, Piantanida E, Lai A. Oxidative stress and Graves' ophthalmopathy: in vitro studies and therapeutic implications. Biofactors 2003;19:155-63.

22. Ventura M, Melo M, Carrilho F. Selenium and thyroid disease: from pathophysiology to treatment. Int $J$ Endocrinol 2017;2017:1297658.

23. Lee YH, Lee SJ, Lee MK, et al. Serum selenium levels in patients with respiratory diseases: a prospective observational study. J Thorac Dis 2016;8:2068-78.

24. Rua RM, Ojeda ML, Nogales F, et al. Serum selenium levels and oxidative balance as differential markers in hepatic damage caused by alcohol. Life Sci 2014;94:158-63.

25. Ravn-Haren G, Bugel S, Krath BN, et al. A short-term intervention trial with selenate, selenium-enriched yeast and selenium-enriched milk: effects on oxidative defence regulation. Br J Nutr 2008;99:883-92.

26. Alfthan G, Xu GL, Tan WH, et al. Selenium supplementation of children in a selenium-deficient area in China: blood selenium levels and glutathione peroxidase activities. Biol Trace Elem Res 2000;73:113-25.

27. Thomson CD, Robinson MF, Butler JA, Whanger PD. Long-term supplementation with selenate and selenomethionine: selenium and glutathione peroxidase (EC 1.11.1.9) in blood components of New Zealand women. Br J Nutr 1993;69:577-88.

28. Combs GF Jr. Selenium in global food systems. Br J Nutr 2001:85:517-47.

29. Stranges S, Marshall JR, Natarajan R, et al. Effects of longterm selenium supplementation on the incidence of type 2 diabetes: a randomized trial. Ann Intern Med 2007;147:21723.

30. Esperidiao-Antonio V, Conceicao-Silva F, De-Ary-Pires B, et al. The human superior tarsal muscle (Müller's muscle): a morphological classification with surgical correlations. Anat Sci Int 2010;85:1-7.

31. Bahn RS. Graves' ophthalmopathy. $N$ Engl J Med 2010;362:726-38.

32. Feldon SE, Levin L. Graves' ophthalmopathy: V. Aetiology of upper eyelid retraction in Graves' ophthalmopathy. $\mathrm{Br} J$ Ophthalmol 1990;74:484-5.

33. Smith TJ, Douglas RS. Pharmacotherapy: does selenium supplementation improve Graves ophthalmopathy? Nat Rev Endocrinol 2011;7:505-6.

34. Campese VM, Ye S, Zhong H, et al. Reactive oxygen species stimulate central and peripheral sympathetic nervous system activity. Am J Physiol Heart Circ Physiol 2004;287:H695-703.

35. Ye S, Zhong H, Yanamadala S, Campese VM. Oxidative stress mediates the stimulation of sympathetic nerve activity in the phenol renal injury model of hypertension. $H y$ pertension 2006;48:309-15. 